\title{
Patterns of renal osteodystrophy one year after kidney transplantation
}

Hanne Skou Jørgensen ${ }^{2}$ (orcid 0000-0002-0881-2615) (Twitter @ hanne_skou), Geert Behets ${ }^{3}$, Bert Bammens $^{14}$ (orcid 0000-0002-2477-2633) (Twitter@mnaesens), Kathleen Claes ${ }^{14}$, Bjorn Meijers ${ }^{14}$ (orcid 0000-0002-2846-2026), Maarten Naesens ${ }^{14}$ (orcid 0000-0002-5625-0792), Ben Sprangers ${ }^{14}$ (orcid 00000003-1314-9675), Dirk RJ Kuypers ${ }^{14}$ (orcid 0000-0001-5546-9680), Patrick D’Haese ${ }^{3}$, Pieter Evenepoel ${ }^{1}$ (orcid 0000-0002-0797-4321) (Twitter@EvenepoelPieter)

${ }^{1}$ Department of Microbiology, Immunology and Transplantation; Nephrology and Renal Transplantation Research Group, KU Leuven, Belgium

${ }^{2}$ Department of Kidney Diseases, Aarhus University Hospital, Aarhus Denmark

${ }^{3}$ Laboratory of Pathophysiology, University of Antwerp, Wilrijk, Belgium

${ }^{4}$ Department of Medicine, Division of Nephrology, University Hospitals Leuven, Belgium

Running head: Renal osteodystrophy after kidney transplantation

Correspondence to: Pieter Evenepoel; E-mail: Pieter.Evenepoel@uzleuven.be 


\begin{abstract}
Background. Renal osteodystrophy is considered common, but is not well characterized, in contemporary kidney transplant recipients. This study reports extensively on bone phenotype by bone histomorphometry, bone densitometry, and novel bone biomarkers 1 year after kidney transplantation.

Methods. A transiliac bone biopsy and dual energy x-ray absorptiometry were performed in 141 unselected kidney transplant recipients in this observational cohort study. Blood and $24 \mathrm{hr}$ urine samples were collected simultaneously.

Results. Median age was $57 \pm 11$ years, $71 \%$ were men, and all were of Caucasian ethnicity. Bone turnover was normal in $71 \%$ of patients, low in $26 \%$, and high in just four cases (3\%). Hyperparathyroidism with hypercalcemia was present in $13 \%$ of patients, of which one had high bone turnover. Delayed bone mineralization was detected in 16\% of patients, who were characterized by hyperparathyroidism (137 vs. 53 $\rho \mathrm{g} / \mathrm{mL})$, a higher fractional excretion of phosphate (40vs. 32\%), and lower levels of phosphate (2.68 vs 3.18 $\mathrm{mg} / \mathrm{dL}$ ) and calcidiol (29vs. $37 \mathrm{ng} / \mathrm{mL}$ ) compared to patients with normal bone mineralization. Osteoporosis was present in $15-46 \%$ of patients, with the highest prevalence at the distal skeleton. The proportion of osteoporotic patients was comparable across categories of bone turnover and mineralization.

Conclusion. The majority of kidney transplant recipients, including patients with osteoporosis, have a normal bone turnover at 1-year post-transplant. Low bone turnover is seen in a substantial subset, while high bone turnover is rare. Vitamin D deficiency and hypophosphatemia represent potential interventional targets to improve bone health post-transplant.
\end{abstract}

Keywords: bone density, bone histomorphometry, chronic kidney disease - mineral and bone disorder, hyperparathyroidism, kidney transplantation

\title{
KEY LEARNING POINTS
}

\section{What is currently known about this subject?}

- Current international guidelines highlight the importance of evaluating bone fragility in kidney transplant recipients, but recommendations for screening and treatment remain vague. 
- To advance treatment strategies for optimal bone health post-transplant, it is essential to characterize the bone phenotype. This study reports extensively on bone disease in an unselected cohort of kidney transplant recipients at 12 months post-transplant, a time-point where kidney function and mineral metabolism is expected to have stabilized.

\section{What this study adds?}

- Normal bone turnover is the most common finding in unselected kidney transplant recipients at 12 months post-transplant. Remaining patients have low bone turnover, which is associated with steroid exposure, while high bone turnover is rare, despite a high prevalence of hyperparathyroidism.

- Bone mineralization is delayed in a subset of patients, and related to disturbances of mineral metabolism with hyperparathyroidism, renal phosphate wasting, and low vitamin D levels.

- Osteoporosis and osteopenia as defined by DXA $T$-scores are highly prevalent in kidney transplant recipients, particularly at the distal skeleton. Importantly, the majority of patients with osteoporosis have normal bone turnover and mineralization at 12 months post-transplant.

\section{What impact this may have on practice or policy?}

- By informing on the bone phenotype of kidney transplant recipients, and particularly the pattern of renal osteodystrophy, we hope to reduce therapeutic nihilism regarding fracture prevention in the post-transplant period. 


\section{INTRODUCTION}

Bone disease after kidney transplantation is an issue of growing concern. ${ }^{1}$ Current international guidelines highlight the importance of evaluating bone fragility in kidney transplant recipients, but recommendations for screening and treatment remain vague. ${ }^{2}$ To advance treatment strategies for optimal bone health posttransplant, it is essential to characterize the bone phenotype.

Pre-transplant renal osteodystrophy, post-transplant immunosuppressive treatment, and ongoing disturbances of mineral metabolism all contribute to bone disease in kidney transplant recipients. The pattern of renal osteodystrophy in patients with kidney failure is changing, shifting from high to low bone turnover states, 3,4 the clinical consequence of which is yet to be established. Immunosuppressive regimens have been optimized, with the introduction of steroid-minimization or steroid-free protocols, contributing to reduced bone loss after transplantation. ${ }^{5,6}$ Changes in mineral metabolism therapy, with introduction of calcimimetics, widespread nutritional vitamin D supplementation, and a more restrictive use of calciumcontaining phosphate binders, may translate to altered mineral metabolism phenotypes post-transplant. ${ }^{7,8}$

The transiliac bone biopsy with subsequent histomorphometric analysis remains the gold standard for evaluating renal osteodystrophy. By the current recommendations, three key histologic features are evaluated, namely bone turnover, mineralization, and volume (TMV classification). ${ }^{9}$ Bone turnover decreases post-transplant, even from a low to normal phenotype pre-transplant. ${ }^{10,11}$ Osteoblast dysfunction was demonstrated in the early post-transplant period and linked to glucocorticoid use and hypophosphatemia. ${ }^{12}$ Mineralization defects are reported to varying degrees. ${ }^{13,14}$

The use of bone biopsies in clinic and research is waning, ${ }^{15}$ and consequently, contemporary studies investigating bone disease after kidney transplantation by histomorphometry are limited. The resulting knowledge-gap, along with the lack of guidance from interventional trials, may lead to therapeutic nihilism, potentially contributing to the high fracture risk seen after kidney transplantation. ${ }^{16,17}$

The aim of this study was to characterize renal osteodystrophy after kidney transplantation by performing protocol-specified bone biopsies at 1 year post-transplant. The contributions of patient demographics, current 
medical treatment, and ongoing disturbances of mineral metabolism to the pattern of renal osteodystrophy in kidney transplant recipients are examined.

\section{MATERIALS AND METHODS}

\section{Cohort}

Adult patients referred for a single kidney transplantation at the University Hospitals Leuven, Belgium were eligible to participate in this prospective, observational study, detailing mineral and bone disorders after kidney transplantation (clinical trial identifier: NCT01886950). The only exclusion criteria was use of antiosteoporotic medications. From $15^{\text {th }}$ October 2010 to $31^{\text {st }}$ August 2018, 955 patients received a single kidney transplant, of which 155 agreed to participate in this study. Eleven patients were excluded from the study due to poor quality of the bone biopsy, and three due to use of anti-resorptive therapy, resulting in a final cohort of 141 patients. Relevant demographic variables, including comorbidities, acute rejections, and medical therapy were extracted from electronic patient files. Patients were scheduled for a study visit with blood samples and a bone biopsy at 12 months post-transplant. The median time from transplant was 376 days [IQR 367 to 385], and $90 \%$ of patients had the bone biopsy performed from 1 week before to 1 month after the 1 year posttransplant date. Study participants were comparable to non-participants, with the exception of being slightly older (57 vs. 55 years, $p=0.04)$ and having higher calcidiol-levels (36 vs. $32 \mathrm{ng} / \mathrm{mL})($ Suppl Table 1).

All clinical and research activities reported are consistent with the Principles of the Declaration of Istanbul on Organ Trafficking and Transplant Tourism. The study adhered to the principles of the Declaration of Helsinki and was approved by the Research Ethical Committee (Study ID S52091) of KU Leuven. All patients provided written informed consent prior to participation.

\section{Immunosuppressive protocol}

Standard triple immunosuppressive therapy with glucocorticoids, a calcineurin inhibitor, and an antimetabolite were initiated at the time of transplantation. Methylprednisolone was administered intravenously on the day of $(500 \mathrm{mg})$ and first day after $(40 \mathrm{mg})$ transplantation, followed by a daily oral dose of $16 \mathrm{mg}$, which was tapered over the first 3 months, at the discretion of the treating physician. Clinical parameters and findings on a protocolled kidney graft biopsy at month 3 determined whether glucocorticoids were discontinued or not. All mineral metabolism therapy was stopped at the time of transplantation and 
resumed as considered necessary by the treating physician. Vitamin D levels were systematically monitored and supplemented to a target level $>30 \mathrm{ng} / \mathrm{mL}$.

\section{Biochemical analyses}

Fasting blood samples were collected at the outpatient study visit in relation to the bone biopsy procedure. Samples were stored for less than 2 hours at $5^{\circ} \mathrm{C}$ before arrival at the laboratory, were they were centrifuged at $3000 \mathrm{rpm}$ for 10 minutes, aliquoted, and stored at $-80^{\circ} \mathrm{C}$ until analysis. $24 \mathrm{hr}$ urine samples were collected, shaken, aliquoted, and stored at $-80^{\circ} \mathrm{C}$ until analysis. Creatinine, hemoglobin, phosphate, total calcium, and bicarbonate were all measured using standard laboratory techniques. The estimated glomerular filtration rate (eGFR) was calculated using the Chronic Kidney Disease Epidemiology Collaboration equation. ${ }^{18}$

Serum 25-hydroxy vitamin D (calcidiol) levels were measured using a radioimmunoassay. ${ }^{19}$ Serum fulllength (biointact) parathyroid hormone (PTH) was determined by an in-house immunoradiometric assay (normal range 3-40 $\rho g / \mathrm{mL}$ ). ${ }^{20}$ Bone-specific alkaline phosphatase (BsAP; normal range 6.1-25.5 ug/L), trimeric procollagen type I N-terminal propeptide (PINP; normal range $12.8-82.6 \mathrm{ng} / \mathrm{mL}$ ), and tartrateresistant acid phosphatase isoform 5b (TRAP5b; normal range 1.1-6.9 U/L) were measured with the ImmunoDiagnostic Systems iSYS instrument (IDS, Boldon, UK). Values above the aupper limit of quantification were determined after dilution. Serum sclerostin (TECOmedical, Sissach, Switzerland; normal range $0.25-0.93 \mathrm{ng} / \mathrm{mL}$ ), osteoprotegerin (OPG) (Biomedica, Vienna, Austria; normal mean: $54 \mathrm{\rho g} / \mathrm{mL}$ ), and soluble receptor activator of nuclear factor kappa-B ligand (sRANKL) (Biomedica, Vienna, Austria; normal range $0.6-9.6 \mathrm{gg} / \mathrm{mL}$ ) were measured using enzyme-linked immunosorbent assay kits as per the manufacturer's instructions. Inter- and intra-assay variation for all assays used were below 10\%. Fractional excretions of phosphate $\left(\mathrm{FEPO}_{4}\right.$; normal range $0-25 \%$ ) and calcium (FECa; normal range 1-2 \%) were calculated based on serum (S) and 24-hour urinary $(U)$ measurements, by the following equations: $\left(\mathrm{U}_{\text {phosphate }}\right.$ $\left.\times \mathrm{S}_{\text {creatinine }}\right) /\left(\mathrm{S}_{\text {phosphate }} \times \mathrm{U}_{\text {creatinine }}\right)$ and $\left(\mathrm{U}_{\text {calcium }} \times \mathrm{S}_{\text {creatinine }}\right) /\left(\mathrm{S}_{\text {calcium }} \times \mathrm{U}_{\text {creatinine }}\right)$, with all measurements in $\mathrm{mg} / \mathrm{dL}$. For the urinary calcium (U-Ca) excretion, $100-250 \mathrm{mg} / 24 \mathrm{hrs}$ was considered the normal range. ${ }^{21}$ 


\begin{abstract}
Bone histomorphometry
A transiliac bone biopsy was performed under light sedation with local anesthesia, using a trephine with an outer/inner diameter of 4.50/3.55 mm (Osteobell 7G [Mirandola, Italy]), at a site $2 \mathrm{~cm}$ posterior and $2 \mathrm{~cm}$ inferior to the anterior iliac spine. Oral tetracyclines were administered twice $(2 \times 500 \mathrm{mg} /$ day for 3 days $)$ with an interval of 11 days, and the bone biopsy performed 4 days after the last intake of tetracycline. Biopsy specimens were fixed in 70\% ethanol and embedded in a methylmethacrylate resin. Un-decalcified 5- $\mu \mathrm{m}$ thick sections were stained by the Goldner method in order to determine static bone parameters, while unstained sections of $10-\mu \mathrm{m}$ thickness were mounted in $100 \%$ glycerol for fluorescence microscopy, to visualize tetracycline labels and determine the dynamic parameters.
\end{abstract}

All histomorphometric parameters are reported in 2D using standardized nomenclature. ${ }^{22}$ The bone histomorphometric analysis was performed at the Laboratory of Pathophysiology, University of Antwerp, Belgium, using a commercially available image analysis program (AxioVision version 4.51, Zeiss Microscopy, Zeiss, Germany) running a custom program. Patients were categorized by the TMV classification ${ }^{9}$ by an experienced bone pathologist, using a semi-quantitative approach, taking into account key dynamic and static parameters. The normal range of the bone formation rate/total tissue area (BFR) was 97-613 $\mu \mathrm{m}^{2} / \mathrm{mm}^{2} /$ day. ${ }^{23}$ Delayed mineralization was defined as the combination of a prolonged mineralization lag time (Mlt, normal range 2-63 days) in combination with high amounts of osteoid (OAr/BAr, normal range $0.2-5.8 \%$ ). Bone volume was considered low if bone on total tissue area (BAr/TAr) was $<14.6 \%$ and high if $\mathrm{BAr} / \mathrm{TAr}$ was $>27.0 \%$.

\title{
Dual energy x-ray absorptiometry
}

Bone mineral density (BMD) was measured by dual-energy x-ray absorptiometry (DXA) using a Hologic densitometer (4500 or Discovery; Hologic, Marlborough, MA, USA). Scans performed \pm 4 weeks from the date of bone biopsy were accepted. A single, certified operator analyzed all DXA scans. The Hologic Spine Phantom was scanned daily to monitor the device performance and long-term stability. $T$ - and $Z$-scores were calculated using reference data from the third National Health and Nutrition Examination Survey, as provided by the manufacturer. 


\section{Statistical analysis}

Data are expressed as mean (SD) or median [IQR]. For comparison of variables across groups, one-way analysis of variance (ANOVA), or the Kruskal-Wallis rank-sum tests were used for normally distributed and skewed variables, respectively. For dichotomous and categorical variables, Pearson's $X^{2}$ or Fisher's exact tests were used. Associations between continuous variables were assessed using Spearman's univariate correlation, with Bonferroni-adjusted $p$ values to account for multiple comparisons. Multivariable linear regression was used to identify independent determinants of key bone histomorphometric variables, applying a stepwise backwards selection with level of $p<0.20$, followed by a forward selection with $p<0.05$. All statistical analyses were performed using STATA IC version 16.1 (StataCorp LP, College Station, TX, USA)

\section{RESULTS}

\section{Demography}

Demographic data including relevant medical therapy are given in Table 1. The majority of patients had received chronic intermittent hemodialysis $(64 \%)$ or continuous peritoneal dialysis $(30 \%)$ prior to kidney transplantation, with a median dialysis vintage of 26 [14-37] months. Immunosuppression was achieved with tacrolimus for nearly all (96\%); a single patient received cyclosporine. Steroids had been halted in $21 \%$ of patients, and current prednisolone dose for the remaining was $2-4 \mathrm{mg}$, with the exception of one patient receiving $10 \mathrm{mg}$. Cumulative steroid dose in the overall cohort amounted to $2.45 \mathrm{~g}$ [1.84-2.93]. Thirty-six patients $(26 \%)$ experienced at least one episode of acute rejection during the first post-transplant year. The cumulative steroid dose in these patients was $4.57 \mathrm{~g}$ [2.47-5.63]. Mean eGFR was $49 \pm 16 \mathrm{ml} / \mathrm{min} / 1.73 \mathrm{~m}^{2} ; 12$ patients had an $\mathrm{eGFR}<30$, and none $<15$. The prevalence of metabolic acidosis, defined as either a serum bicarbonate $<20 \mathrm{mmol} / \mathrm{L}$ or the use of oral bicarbonate supplement, was $41 \%$.

\section{Renal osteodystrophy}

Distribution of bone turnover, mineralization, and volume at 12 months post-transplant are shown in Figure 1, and patient characteristics according to the TMV classification are given in Table 2. Bone turnover was normal in the majority (71\%), with low turnover in $26 \%$, and high turnover in $3 \%$ of patients. Patients with low bone turnover had received a $22 \%$ higher cumulative dose of steroids compared to those with normal bone turnover. There was a trend towards lower levels of calcidiol in patients with high turnover. The 
biochemical bone turnover markers, (BsAP, Intact PINP, and TRAP5b were significantly lower in patients with low bone turnover, with numerically higher levels in patients with high turnover.

Delayed mineralization was seen in $16 \%$ of patients, none of which had overt osteomalacia. Patients with delayed mineralization were younger and more obese, and had received a $26 \%$ higher cumulative dose of steroids. Delayed mineralization was associated with higher levels of biointact PTH, a higher $24 \mathrm{hr}$ urinary $\mathrm{FEPO}_{4}$, and lower levels of phosphate, calcidiol, magnesium, bicarbonate, and sclerostin.

Bone volume was low in $18 \%$ of patients, who tended to be older, but otherwise had comparable demography to that of patients with normal bone volume. sRANKL levels were lower, with a trend towards higher OPG levels, and consequently the OPG/sRANKL-ratio was significantly higher (median 51 vs. 34, $p$ $=0.003$ ) in patients with low bone volume.

\section{Bone densitometry}

A DXA-scan was available for 108 patients (77\%) at the lumbar spine, $106(75 \%)$ at the proximal femur, and $91(65 \%)$ at the distal forearm. The prevalence of osteoporosis and osteopenia, as defined by DXA $T$-scores, at different skeletal sites are shown in Figure 2. Osteoporosis prevalence was comparable across categories of bone turnover, and between patients with and without delayed mineralization (Table 3). The diagnostic overlap between low bone volume on bone biopsy vs. low BMD by DXA was incomplete; only $33 \%$ of patients with DXA-defined osteoporosis at the femoral neck were categorized as having low bone volume by bone histomorphometry.

\section{Mineral metabolism}

Only 44 patients (31\%) had all biochemical parameters of mineral metabolism within the target range. Hyperparathyroidism (biointact PTH $>1.5$ times above the normal range) was the most prevalent disturbance (47\%), while vitamin D insufficiency $(<30 \mathrm{ng} / \mathrm{mL})$ was seen in $38 \%$, hypercalcemia $(\mathrm{tCA}>10.3 \mathrm{mg} / \mathrm{dL})$ in $18 \%$, and hypophosphatemia $(<2.3 \mathrm{mg} / \mathrm{dl})$ in $13 \%$ of patients. Vitamin D deficiency $(<15 \mathrm{ng} / \mathrm{mL})$ was detected in just $4 \%$. There was a noticeable overlap between these categories (Figure 3).

Histomorphometric findings in patients with disturbed mineral metabolism are given in Table 4. Hyperparathyroidism was associated with higher bone turnover, while this was not the case for 
hypercalcemia. Patients with hypophosphatemia had greater amounts of osteoid, and were more likely to have delayed mineralization ( $39 v s .12 \%, p=0.004$ ), as compared to patients with normal phosphate levels. In accordance with these findings, biointact PTH was directly correlated to all parameters of skeletal remodeling, while phosphate level correlated inversely with parameters of mineralization (Suppl Table 2).

\section{Determinants of bone turnover, mineralization, and volume}

To identify independent determinants of TMV status, we performed multivariable linear regression analyses, with key bone histomorphometric parameters as the outcome, and a stepwise backwards selection of explanatory variables, including demographics, post-transplant kidney function, cumulative steroid dose, and biochemical markers of mineral metabolism. Results of univariate analyses are given in Suppl Table 3.

Independent determinants of bone turnover (BFR) were PTH $(\beta=0.684, p<0.001)$ and cumulative steroid dose $\left(\beta=-0.218, p=0.02\right.$; model adjusted $\left.R^{2} 16 \%\right)$. Determinants of mineralization (Mlt) were phosphate $(\beta$ $=-0.481, p=0.001)$ and cumulative steroid dose $\left(\beta=0.182, p=0.004\right.$; model adjusted $\left.R^{2} 15 \%\right)$, and determinants of bone volume (BAr/TAr) were age $(\beta=-0.150, p=0.002)$, and $\operatorname{BMI}(\beta=0.331, p=0.002$; model adjusted $\left.R^{2} 11 \%\right)$.

\section{DISCUSSION}

The key findings of this study detailing bone phenotype at 1 year post-transplant are that (i) bone turnover is normal in the majority of kidney transplant recipients; (ii) high bone turnover is rare, despite a high prevalence of hyperparathyroidism; and (iii) delayed bone mineralization is seen in a subset of patients, and is associated with disturbed mineral metabolism.

\section{Bone turnover}

Bone turnover was low or normal in $97 \%$ of patients at 1 year post-transplant. This should be seen in the context of the current pattern of renal osteodystrophy pre-transplant, where low bone turnover is reported in $60-80 \%$ of Caucasian patients with kidney failure. ${ }^{4,24}$ Recent studies in kidney transplant recipients similarly report mainly low (26-64\%) or normal (17-48\%) bone turnover, with the prevalence of high turnover in the range of $0-19 \% .^{10,11,14,25,26}$ Variation between studies may be explained by heterogeneity of patient characteristics, particularly time since transplantation, as well as differences in diagnostic criteria of 
histomorphometry. As bone biopsies were performed not on indication, but per research protocol, our findings should be representative of the current pattern of renal osteodystrophy in kidney transplant recipients.

The clinical consequence of low bone turnover post-transplant is unknown. Medically induced low bone turnover is the hallmark of osteoporosis treatment, aiming to reduce bone loss by inhibiting bone resorption. In the setting of $\mathrm{CKD}$, however, low bone turnover is characterized by reductions in both bone formation and resorption, a combination that may negatively impact bone mechanical properties. ${ }^{27}$ In patients with kidney failure, low bone turnover associates with older age and a greater burden of comorbidity, ${ }^{28}$ representing important confounders for the clinical implications of this phenotype.

Determinants of BFR, the key histomorphometric parameter of bone turnover, were current PTH level and cumulative steroid dose. The direct association with PTH is consistent with our understanding of the effects of hyperparathyroidism on skeletal remodeling. The inverse relationship between bone turnover and steroids is also well known, ${ }^{12,13}$ as a decreased bone formation due to inhibition of osteoblast function is the key mechanism behind corticosteroid-related bone loss..$^{29}$

High bone turnover was a rare finding one year post-transplant, observed in just $3 \%$ of patients. This low occurrence, despite a high prevalence of hyperparathyroidism, could imply continued skeletal resistance to PTH in kidney transplant recipients. ${ }^{30}$ We used a cutoff of 1.5 times the upper limit of the normal range to define hyperparathyroidism, but there is no clear recommendation on what PTH range to target posttransplant. ${ }^{2}$ Indeed, if PTH hyporesponsiveness remains an issue post-transplant, the optimal range of PTH may vary depending on kidney function.

Hyperparathyroidism with hypercalcemia was not indicative of high bone turnover, supporting previous reports. ${ }^{10,31}$ Hypercalcemia was also not associated with elevated histomorphometric parameters of skeletal remodeling, suggesting that mechanisms other than skeletal efflux of calcium, i.e. increased gastro-intestinal uptake or renal calcium reabsorption, are involved in hypercalcemia post-transplant. The latter seems likely, as the urinary calcium excretion was generally low. Both hyperparathyroidism and rising levels of calcitriol 
may contribute to increased renal calcium reabsorption post-transplant. ${ }^{32}$ Additional studies are required to clarify the pathophysiology as well as the consequence of hypercalcemia in kidney transplant recipients.

\section{Bone mineralization}

Delayed mineralization was present in $16 \%$ of patients in this cohort. Previous studies report mineralization defects in the range of $22-88 \%$, with no clear trends depending on the time since transplantation. ${ }^{10,11,13,14,26}$ Several definitions are currently in use to describe abnormal mineralization in renal osteodystrophy, rendering comparisons difficult. We used a semiquantitative approach, defining delayed mineralization as prolonged mineralization lag time (Mlt $>50$ days), with increased amounts of osteoid either focally or globally. None of our patients had overt osteomalacia, a phenotype that has become rare after the disappearance of aluminum bone disease. ${ }^{33}$

Low levels of phosphate, calcidiol, bicarbonate, and sclerostin were associated with delayed mineralization. Adequate phosphate and vitamin D levels are necessary for normal biomineralization. ${ }^{34}$ While vitamin D levels were lower in patients with delayed mineralization, calcidiol was not an independent predictor of Mlt, and did not correlate with histomorphometric parameters. Two previous studies failed to find an association between calcidiol and bone mineralization in kidney transplant recipients. ${ }^{13,14}$ Hypophosphatemia has been implicated in osteoblast apoptosis in the early post-transplant period, ${ }^{12}$ and similar to our findings, lower phosphate levels were reported in patients with delayed mineralization $>2$ years after kidney transplantation. ${ }^{14}$ A normal physiological response to hypophosphatemia is to increase renal phosphate reabsorption, virtually abolishing phosphate in the urine, but kidney transplant recipients have a high urinary phosphate excretion, ${ }^{31}$ likely due to effects of ongoing hyperparathyroidism and hyperphosphatoninism on the kidney graft. ${ }^{35,36}$ Consistent with this, our 24-hour urinary analysis revealed a high fractional excretion of phosphate in this cohort, and particularly so in patients with delayed bone mineralization. Although low levels of phosphate associate with better long-term outcomes posts-transplant, with reduced risk of graft failure and cardiovascular events; ${ }^{37}$ our findings highlight potential harmful effects in bone. In line with this, hypophosphatemia was associated with fractures in a retrospective analysis of kidney transplant recipients. ${ }^{38}$ 


\begin{abstract}
Metabolic acidosis leads to bone demineralization as excess protons are buffered through dissolution of skeletal calcium-carbonate, ${ }^{39}$ and may also inhibit osteoblast differentiation and function ${ }^{40}$. The potential effect of bicarbonate supplementation on bone quality in CKD is poorly investigated. Metabolic acidosis associates with low BMD in kidney transplant recipients, ${ }^{41}$ and correction improved bone microarchitecture in one study which included histomorphometry; unfortunately, bone mineralization was not reported. ${ }^{42}$ Lastly, sclerostin level was $26 \%$ lower in patients with delayed mineralization. Although a potential role of sclerostin in bone mineralization has been proposed, ${ }^{43}$ this finding may also reflect the inverse relation between sclerostin and PTH levels. ${ }^{44}$
\end{abstract}

\title{
Bone volume
}

Bone volume was low in $19 \%$ of patients at 1 year post-transplant, as evaluated by the bone biopsy. Previous studies report higher prevalence of 26-56\%, generally increasing with time since kidney transplantation. ${ }^{11,13,14}$ Patients with low bone volume had higher levels of OPG - a counterintuitive finding considering that the role of OPG in bone remodeling is to inhibit osteoclast differentiation. Nevertheless, an inverse relationship between OPG levels and BMD was previously reported in patients with CKD. ${ }^{45,46}$ As OPG and sRANKL are effectors of bone metabolism, their relationship with bone mass is likely complex. The only independent determinants of bone volume in this cohort were age and BMI, which explained just $11 \%$ of the variation in BAr/TAr. Cumulative steroid dose was not correlated to bone volume, in contrast to previous reports. ${ }^{10,13}$ However, as this was a cross-sectional study, we could not evaluate the effect of steroid exposure on bone loss.

Osteoporosis as defined by DXA $T$-scores was present in $15-46 \%$ of patients, with the highest prevalence at the distal skeleton. The overlap between low bone volume by bone biopsy vs. low BMD by DXA was not impressive, as two-thirds of patients with DXA-defined osteoporosis at the femoral neck were classified as having normal bone volume by bone histomorphometry. Previous studies similarly report moderate correlations at most between bone volume by bone biopsy and BMD by DXA. ${ }^{10,11}$ Bone histomorphometry is likely the less suitable method for assessing bone mass, as the sample size is small, and findings are specific for the site of the bone biopsy, i.e. the iliac crest. In contrast, DXA BMD captures a much larger area of 
bone, and can be measured at several different skeletal sites. DXA BMD is rapidly gaining acceptance as a clinically relevant measure of bone fragility in $\mathrm{CKD},{ }^{2,47}$ although, as an important limitation, it cannot inform on disturbances of skeletal remodeling and mineralization, which are the hallmarks of renal osteodystrophy.

There was no difference in the prevalence of osteoporosis across categories of bone turnover, nor between patients with or without delayed mineralization. The majority of patients with DXA-defined osteoporosis, irrespective of skeletal site, had normal bone biopsy findings, which may to some degree alleviate fears of exacerbating low turnover states or disturbed mineralization when considering anti-resorptive therapy aimed at reducing fracture risk post-transplant. Biochemical markers of skeletal remodeling could potentially aid such treatment decisions; levels of BsAP, Intact PINP, and TRAP5b were all significantly lower in patients with low bone turnover, and have previously been shown to discriminate bone turnover in patients with kidney failure. ${ }^{48}$ As these biomarkers do not accumulate in $\mathrm{CKD},{ }^{49}$ they may be particularly suitable in the post-transplant setting; however, this remains to be investigated.

\section{Strengths and limitations}

Strengths of this study include the substantial number of bone biopsies, the unselected nature of the cohort, and the standardized post-transplant window. Kidney graft function, immunosuppression, and mineral metabolism all stabilize around 12 weeks post-transplant, leaving 9 months for the bone to adapt to the altered metabolic environment. An experienced bone pathologist blinded to patient characteristics performed a full quantitative histomorphometric analysis. The cross-sectional and single-center design may be considered the main limitation. All participants were Caucasian, which may limit generalizability to cohorts of other ethnicities. We report total calcium levels, which have been shown to underestimate hypercalcemia. ${ }^{50}$ Despite these limitations, this is to our knowledge the largest study evaluating renal osteodystrophy by bone histomorphometry in kidney transplant recipients to date, and as patients were unselected, results should be representative of this patient population. There is a lack of consensus on diagnostic cutoffs defining the lesions of renal osteodystrophy, and several different normative references are currently in use. Applying different diagnostic cutoffs would affect the prevalence of disturbed bone turnover and mineralization. Achieving a broad consensus on the applied methodology, appropriate reference ranges, 
and diagnostic cutoffs of bone histomorphometry for the diagnosis of renal ostodystrophy is an urgent priority. ${ }^{15}$

\section{Conclusions and future aspects}

In conclusion, bone turnover is low to normal in the majority of kidney transplant recipients at 1 year posttransplant. High bone turnover is a rare finding, despite persistent hyperparathyroidism, suggesting that PTH is a poor guide for treatment decisions. While a bone biopsy remains the gold standard to evaluate bone turnover, it is both invasive and time-consuming, and non-invasive alternatives are clearly needed. Promising results have been shown for novel biochemical markers of skeletal remodeling, although diagnostic accuracy studies are needed to validate these biomarkers in the post-transplant setting. Importantly, the clinical implications of the different renal osteodystrophy phenotypes post-transplant are largely unknown and deserves further investigation. Collaborative efforts like the European Renal Osteodystrophy (EUROD) initiative $\mathrm{e}^{15}$ may be expected to deliver robust outcome data in the future.

\section{ACKNOWLEDGEMENTS}

HSJ was supported by an ERA-EDTA long-term fellowship in the CKD-MBD working group, and received additional funding from the Augustinus Foundation and Kornings Fund. The authors would like to acknowledge the excellent technical support of Marc Dekens, Henriette de Loor, Albert Herelixka and Herman Borghs at KU/UZ Leuven, and to thank the centers of the Leuven Collaborative Group for Renal Transplantation, the clinicians and surgeons, nursing staff, and the patients who participated in this study.

\section{CONFLICT OF INTEREST STATEMENT}

BB reports grants and other support from Otsuka Pharmaceutical, and other support from Baxter, outside the submitted work. KC reports other support from Astellas, Astra Zeneca, and Alexion, outside the submitted work. BM reports grants and personal fees from Nipro and personal fees from Astra Zeneca, Baxter, and Bayer, outside the submitted work. PE reports personal and other fees from Amgen, outside the submitted work. BS and MN are senior clinical investigators of The Research Foundation Flanders (1842919N and 1844019N, respectively). Remaining authors (DK, GB, HSJ, PDH) report no conflicts of interest. 


\begin{abstract}
AUTHORS' CONTRIBUTIONS
PE was responsible for the conception and design of the study. HSJ performed data analyses, and drafted the first version of the article. All authors provided intellectual content of importance to the performance of the study, contributed to the analysis and interpretation of the data, and critically revised the manuscript.

\section{FUNDING}

HSJ was supported by an ERA-EDTA long-term fellowship in the CKD-MBD working group, and received financial support from the Augustinus Foundation and Kornings Fund at the time of this study.

\title{
DATA AVAILABILITY STATEMENT
}

The data underlying this article cannot be shared publicly due to the privacy of individuals that participated in the study. The data will be shared on reasonable request to the corresponding author.

\section{REFERENCES}

1. Naylor KL, Zou G, Leslie WD, et al. Risk factors for fracture in adult kidney transplant recipients. World J Transplant. 2016;6(2):370-379. doi:10.5500/wjt.v6.i2.370

2. Kidney Disease: Improving Global Outcomes (KDIGO) CKD-MBD Update Work Group. KDIGO 2017 Clinical Practice Guideline Update for the Diagnosis, Evaluation, Prevention, and Treatment of Chronic Kidney Disease-Mineral and Bone Disorder (CKD-MBD). Kidney Int Suppl. 2017;7(1):159. doi:10.1016/j.kisu.2017.04.001

3. Cejka D, Herberth J, Branscum AJ, et al. Sclerostin and Dickkopf-1 in renal osteodystrophy. Clin J Am Soc Nephrol. 2011;6(4):877-882. doi:10.2215/CJN.06550810; 10.2215/CJN.06550810

4. Sprague SM, Bellorin-Font E, Jorgetti V, et al. Diagnostic Accuracy of Bone Turnover Markers and Bone Histology in Patients With CKD Treated by Dialysis. Am J Kidney Dis. 2016;67(4):559-566. doi:10.1053/j.ajkd.2015.06.023

5. Nikkel LE, Mohan S, Zhang A, et al. Reduced fracture risk with early corticosteroid withdrawal after kidney transplant. Am J Transplant. 2012;12(3):649-659. doi:10.1111/j.1600-6143.2011.03872.x; 10.1111/j.1600-6143.2011.03872.x

6. Iyer SP, Nikkel LE, Nishiyama KK, et al. Kidney transplantation with early corticosteroid 
withdrawal: paradoxical effects at the central and peripheral skeleton. J Am Soc Nephrol. 2014;25(6):1331-1341. doi:10.1681/ASN.2013080851

7. Evenepoel P, Sprangers B, Lerut E, et al. Mineral metabolism in renal transplant recipients discontinuing cinacalcet at the time of transplantation: a prospective observational study. Clin Transplant. 2012;26(3):393-402. doi:10.1111/j.1399-0012.2011.01524.x

8. Perrin P, Kiener C, Javier R-M, et al. Recent Changes in Chronic Kidney Disease-Mineral and Bone Disorders and Associated Fractures After Kidney Transplantation. Transplantation. 2017;101(8):1897-1905. doi:10.1097/TP.0000000000001449

9. Moe S, Drüeke T, Cunningham J, et al. Definition, evaluation, and classification of renal osteodystrophy: a position statement from Kidney Disease: Improving Global Outcomes (KDIGO). Kidney Int. 2006;69(11):1945-1953. doi:10.1038/sj.ki.5000414

10. Evenepoel P, Behets GJ, Viaene L, D'Haese PC. Bone histomorphometry in de novo renal transplant recipients indicates a further decline in bone resorption 1 year posttransplantation. Kidney Int. 2017;91(2):469-476. doi:10.1016/j.kint.2016.10.008

11. Keronen S, Martola L, Finne P, Burton IS, Kröger H, Honkanen E. Changes in bone histomorphometry after kidney transplantation. Clin J Am Soc Nephrol. 2019;14(6):894-903. doi:10.2215/CJN.09950818

12. Rojas E, Carlini RG, Clesca P, et al. The pathogenesis of osteodystrophy after renal transplantation as detected by early alterations in bone remodeling. Kidney Int. 2003;63(5):1915-1923. doi:10.1046/j.1523-1755.2003.00938.x

13. Monier-Faugere MC, Mawad H, Qi Q, Friedler RM, Malluche HH. High prevalence of low bone turnover and occurrence of osteomalacia after kidney transplantation. J Am Soc Nephrol. 2000;11(6):1093-1099. doi:DOI: 10.1681/ASN.V1161093

14. Neves CL, Dos Reis LM, Batista DG, et al. Persistence of bone and mineral disorders 2 years after 
successful kidney transplantation. Transplantation. 2013;96(3):290-296.

doi:10.1097/TP.0b013e3182985468

15. Evenepoel P, D'Haese P, Bacchetta J, et al. Bone biopsy practice patterns across Europe: the European renal osteodystrophy initiative-a position paper. Nephrol Dial Transplant. 2017;32(10):1608-1613. doi:10.1093/ndt/gfw468

16. Hansen D, Olesen JB, Gislason GH, Abrahamsen B, Hommel K. Risk of fracture in adults on renal replacement therapy: a Danish national cohort study. Nephrol Dial Transplant. 2016;31(10):16541662. doi:10.1093/ndt/gfw073

17. Nikkel LE, Hollenbeak CS, Fox EJ, Uemura T, Ghahramani N. Risk of fractures after renal transplantation in the United States. Transplantation. 2009;87(12):1846-1851. doi:10.1097/TP.0b013e3181a6bbda

18. Inker LA, Schmid CH, Tighiouart H, et al. Estimating Glomerular Filtration Rate from Serum Creatinine and Cystatin C. N Engl J Med. 2012;367(1):20-29. doi:10.1056/NEJMoa1114248

19. Bouillon R, Van Herck E, Jans I, Tan BK, Van Baelen H, De Moor P. Two direct (nonchromatographic) assays for 25-hydroxyvitamin D. Clin Chem. 1984;30(11):1731-1736.

20. Bouillon R, Coopmans W, Degroote DE, Radoux D, Eliard PH. Immunoradiometric assay of parathyrin with polyclonal and monoclonal region-specific antibodies. Clin Chem. 1990;36(2):271276.

21. Curhan GC, Willett WC, Speizer FE, Stampfer MJ. Twenty-four-hour urine chemistries and the risk of kidney stones among women and men. Kidney Int. 2001;59(6):2290-2298. doi:10.1046/j.15231755.2001.00746.x

22. Dempster DW, Compston JE, Drezner MK, et al. Standardized nomenclature, symbols, and units for bone histomorphometry: A 2012 update of the report of the ASBMR Histomorphometry Nomenclature Committee. J Bone Miner Res. 2013;28(1):2-17. doi:10.1002/jbmr.1805 
23. Salusky IB, Coburn JW, Brill J, et al. Bone diseae in pediatric patients undergoing dialysis with CAPD or CCPD. Kidney Int. 1988;33(5):975-982. doi:10.1038/ki.1988.96

24. Malluche HH, Mawad HW, Monier-Faugere M-C. Renal osteodystrophy in the first decade of the new millennium: analysis of 630 bone biopsies in black and white patients. J Bone Miner Res. 2011;26(6):1368-1376. doi:10.1002/jbmr.309

25. Coco M, Pullman J, Cohen HW, et al. Effect of risedronate on bone in renal transplant recipients. $J$ Am Soc Nephrol. 2012;23(8):1426-1437. doi:10.1681/ASN.2011060623

26. Marques IDB, Araújo MJCLN, Graciolli FG, et al. A randomized trial of zoledronic acid to prevent bone loss in the first year after kidney transplantation. J Am Soc Nephrol. 2019;30(2):355-365. doi:10.1681/ASN.2018060656

27. $\mathrm{Ng} \mathrm{AH}$, Omelon S, Variola F, et al. Adynamic Bone Decreases Bone Toughness During Aging by Affecting Mineral and Matrix. J Bone Miner Res. 2016;31(2):369-379. doi:10.1002/jbmr.2702

28. de Oliveira RA, Barreto FC, Mendes M, et al. Peritoneal dialysis per se is a risk factor for sclerostinassociated adynamic bone disease. Kidney Int. 2015;87(5):1039-1045. doi:10.1038/ki.2014.372 [doi]

29. Rizzoli R, Biver E. Glucocorticoid-induced osteoporosis: Who to treat with what agent? Nat Rev Rheumatol. 2015;11(2):98-109. doi:10.1038/nrrheum.2014.188

30. Evenepoel P, Bover J, Ureña Torres P. Parathyroid hormone metabolism and signaling in health and chronic kidney disease. Kidney Int. 2016;90(6):1184-1190. doi:10.1016/j.kint.2016.06.041

31. Borchhardt K, Sulzbacher I, Benesch T, Födinger M, Sunder-Plassmann G, Haas M. Low-turnover bone disease in hypercalcemic hyperparathyroidism after kidney transplantation. Am J Transplant. 2007;7(11):2515-2521. doi:10.1111/j.1600-6143.2007.01950.x

32. Evenepoel P, Van Den Bergh B, Naesens M, et al. Calcium metabolism in the early posttransplantation period. Clin J Am Soc Nephrol. 2009;4(3):665-672. doi:10.2215/CJN.03920808 
33. Malluche HH, Mawad HW, Monier-Faugere MC. Renal osteodystrophy in the first decade of the new millennium: Analysis of 630 bone biopsies in black and white patients. J Bone Miner Res. 2011;26(6):1368-1376. doi:10.1002/jbmr.309

34. Bhan A, Qiu S, Rao SD. Bone histomorphometry in the evaluation of osteomalacia. Bone Reports. 2018;8:125-134. doi:10.1016/j.bonr.2018.03.005

35. Evenepoel P, Naesens M, Claes K, Kuypers D, Vanrenterghem Y. Tertiary “hyperphosphatoninism” accentuates hypophosphatemia and suppresses calcitriol levels in renal transplant recipients. Am J Transplant. 2007;7(5):1193-1200. doi:10.1111/j.1600-6143.2007.01753.x

36. Sirilak S, Chatsrisak K, Ingsathit A, et al. Renal phosphate loss in long-term kidney transplantation. Clin J Am Soc Nephrol. 2012;7(2):323-331. doi:10.2215/CJN.06380611

37. Van Londen M, M. Aarts B, Deetman PE, et al. Post-transplant hypophosphatemia and the risk of death-censored graft failure and mortality after kidney transplantation. Clin J Am Soc Nephrol. 2017;12(8):1301-1310. doi:10.2215/CJN.10270916

38. Aleksova J, Wong P, Mulley WR, et al. Serum phosphorus levels and fracture following renal transplantation. Clin Endocrinol (Oxf). 2017;87(2):141-148. doi:10.1111/cen.13363

39. Bushinsky DA, Lechleider RJ. Mechanism of proton-induced bone calcium release: calcium carbonate-dissolution. Am J Physiol Physiol. 1987;253(5):F998-F1005. doi:10.1152/ajprenal.1987.253.5.F998

40. Takeuchi S, Hirukawa K, Togari A. Acidosis Inhibits Mineralization in Human Osteoblasts. Calcif Tissue Int. 2013;93(3):233-240. doi:10.1007/s00223-013-9746-2

41. Heaf J, Tvedegaard E, Kanstrup I-L, Fogh-Andersen N. Bone loss after renal transplantation: role of hyperparathyroidism, acidosis, cyclosporine and systemic disease. Clin Transplant. 2000;14(5):457463. doi:10.1034/j.1399-0012.2000.140503.x

42. Starke A, Corsenca A, Kohler T, et al. Correction of metabolic acidosis with potassium citrate in 
43. Hassler N, Roschger A, Gamsjaeger S, et al. Sclerostin Deficiency Is Linked to Altered Bone Composition. J Bone Miner Res. 2014;29(10):2144-2151. doi:10.1002/jbmr.2259

44. Evenepoel P, Claes K, Viaene L, et al. Decreased Circulating Sclerostin Levels in Renal Transplant Recipients With Persistent Hyperparathyroidism. Transplantation. 2016;100(10):2188-2193. doi:10.1097/TP.0000000000001311

45. Huang JW, Famure O, Li Y, Kim SJ. Hypomagnesemia and the Risk of New-Onset Diabetes Mellitus after Kidney Transplantation. J Am Soc Nephrol. 2016;27(6):1793-1800.

doi:10.1681/ASN.2015040391

46. Evenepoel P, Claes K, Meijers B, et al. Bone mineral density, bone turnover markers, and incident fractures in de novo kidney transplant recipients. Kidney Int. 2019;95(6):1461-1470. doi:10.1016/j.kint.2018.12.024

47. Evenepoel P, Cunningham J, Ferrari S, et al. European Consensus Statement on the diagnosis and management of osteoporosis in chronic kidney disease stages G4-G5D. Nephrol Dial Transplant. 2021;36(1):42-59. doi:10.1093/ndt/gfaa192

48. Salam S, Gallagher O, Gossiel F, Paggiosi M, Khwaja A, Eastell R. Diagnostic accuracy of biomarkers and imaging for bone turnover in renal osteodystrophy. J Am Soc Nephrol. 2018;29(5):1557-1565. doi:10.1681/ASN.2017050584

49. Evenepoel P, Cavalier E, D’Haese PC. Bone biomarkers in de novo renal transplant recipients. Clin Chim Acta. 2020;501(September 2019):179-185. doi:10.1016/j.cca.2019.10.035

50. Evenepoel P, Bammens B, Claes K, Kuypers D, Meijers BKI, Vanrenterghem Y. Measuring total blood calcium displays a low sensitivity for the diagnosis of hypercalcemia in incident renal transplant recipients. Clin J Am Soc Nephrol. 2010;5(11):2085-2092. doi:10.2215/CJN.02460310 


\section{TABLES}

Table 1. Demographic data at 12 months post kidney transplantation

\begin{tabular}{|c|c|}
\hline & All patients $(n=141)$ \\
\hline Age, yrs & $57 \pm 11$ \\
\hline Gender, male $(\%)$ & $100(71 \%)$ \\
\hline Body mass index, $\mathrm{kg} / \mathrm{m}^{2}$ & $25.3 \pm 4.9$ \\
\hline Dialysis vintage, mo & $26[14,37]$ \\
\hline Diabetes mellitus type $1(\%)$ & $5(3.5 \%)$ \\
\hline Diabetes mellitus type $2(\%)$ & $42(30 \%)$ \\
\hline Previous parathyroidectomy (\%) & $15(11 \%)$ \\
\hline Previous fracture $(\%)$ & $14(10 \%)$ \\
\hline \multicolumn{2}{|l|}{ Cause of kidney failure } \\
\hline Cystic/hereditary/congenital diseases & $34(24 \%)$ \\
\hline Glomerulonephritis/vasculitis & $33(23 \%)$ \\
\hline Diabetic nephropathy & $14(10 \%)$ \\
\hline Hypertensive/large vessel disease & $14(10 \%)$ \\
\hline Interstitial nephritis & $8(6 \%)$ \\
\hline Miscellaneous & $6(4 \%)$ \\
\hline Unknown & $32(23 \%)$ \\
\hline $\mathrm{eGFR}, \mathrm{ml} / \mathrm{min} / 1.73 \mathrm{~m}^{2}$ & $49 \pm 16$ \\
\hline Biointact PTH, $\rho g / m L$ (normal range 3-40) & $56[39,109]$ \\
\hline Phosphate, mg/dL & $3.09 \pm 0.71$ \\
\hline Total calcium, mg/dL & $9.74 \pm 0.88$ \\
\hline Calcidiol, $\mathrm{ng} / \mathrm{mL}$ & $36 \pm 14$ \\
\hline Bicarbonate, $\mathrm{mmol} / \mathrm{L}$ & $23.0 \pm 2.9$ \\
\hline $24 \mathrm{hr}$ U-calcium, mg & $90[43,141]$ \\
\hline $24 \mathrm{hr}$ U-FECa, \% (normal 1-2\%) & $1.0[0.6,1.5]$ \\
\hline 24 hr U-phosphate, mg & $846[663,1106]$ \\
\hline $24 \mathrm{hr} \mathrm{U-FEPO}{ }_{4} \cdot \%$ (normal 0-25\%) & $33[24,40]$ \\
\hline \multicolumn{2}{|l|}{ Current medical therapy } \\
\hline Prednisolone $(\%)$ & $111(79 \%)$ \\
\hline Calcineurin inhibitor $(\%)$ & $137(97 \%)$ \\
\hline Antimetabolite (\%) & $122(87 \%)$ \\
\hline Calcium-supplement (\%) & $33(23 \%)$ \\
\hline Nutritional vitamin D (\%) & $58(41 \%)$ \\
\hline Active vitamin D (\%) & $19(13 \%)$ \\
\hline Cinacalcet $(\%)$ & $1(0.7 \%)$ \\
\hline Bicarbonate supplement (\%) & $50(35 \%)$ \\
\hline
\end{tabular}

Data are mean $\pm \mathrm{SD}$, median [IQR], or $n(\%)$

Abbr.: eGFR=estimated glomerular filtration rate, $\mathrm{FECa}=$ fractional excretion of calcium, $\mathrm{FEPO}_{4}=$ fractional excretion of phosphate, $\mathrm{PTH}=$ parathyroid hormone 
Table 2. Patient characteristics based on classification of bone turnover, mineralization, and volume

\section{Turnover}

\begin{tabular}{|c|c|c|c|c|c|c|}
\hline $\operatorname{Low}(n=37)$ & Normal $(n=100)$ & High $(n=4)$ & Normal $(n=119)$ & Delayed (n=22) & Normal $(n=114)$ & Low $(n=27)$ \\
\hline $60 \pm 11$ & $56 \pm 11$ & $55 \pm 12$ & $58 \pm 11$ & $52 \pm 11 \Phi$ & $56 \pm 12$ & $61 \pm 9 *$ \\
\hline $29(78 \%)$ & $68(68 \%)$ & $3(75 \%)$ & $84(71 \%)$ & $16(73 \%)$ & $78(68 \%)$ & $22(82 \%)$ \\
\hline $25.7 \pm 4.9$ & $25.3 \pm 5.0$ & $22.9 \pm 2.8$ & $24.8 \pm 4.3$ & $28.0 \pm 7.2 \Phi$ & $25.5 \pm 5.0$ & $24.6 \pm 4.8$ \\
\hline $29[19,36]$ & $25[13,38]$ & $29[9,56]$ & $27[13,40]$ & $19[14,29]$ & $26[16,35]$ & $30[15,57]$ \\
\hline $14(38 \%)$ & $32(32 \%)$ & $1(25 \%)$ & $43(36 \%)$ & $4(18 \%)$ & $38(33 \%)$ & $9(33 \%)$ \\
\hline $2.78[1.97,4.88] \mathrm{T}$ & $2.39[1.80,2.85]$ & $1.70[1.39,2.74]$ & $2.36[1.80,2.89]$ & $2.85[2.49,4.80] \mathrm{T}$ & $2.44[1.80,2.94]$ & $2.45[1.97,2.94]$ \\
\hline $48 \pm 15$ & $50 \pm 16$ & $47 \pm 8$ & $50 \pm 16$ & $46 \pm 14$ & $50 \pm 16$ & $47 \pm 13$ \\
\hline $53.5[42.4,78.7]$ & $60.0[38.4,115]$ & $124[22.9,342]$ & $53.0[34.5,89.2]$ & $137[66.7,218] \Phi$ & $56.1[39.3,110]$ & $64.4[38.9,93.9]$ \\
\hline $1.3[1.1,2.0]$ & $1.5[1.0,2.9]$ & $3.1[0.7,7.5]$ & $1.3[0.9,2.2]$ & $3.4[1.7,5.5] \mp$ & $1.4[1.0,2.8]$ & $1.1[0.6,1.4]$ \\
\hline $68[52 ; 87]^{*}$ & $80[63 ; 95]$ & $111[74 ; 243] \Phi$ & $77[58 ; 91]$ & $84[67 ; 102]$ & $82[61 ; 115]$ & $76[58 ; 92]$ \\
\hline $16.7[11.5 ; 23.7] \mathrm{T}$ & $21.9[14.4 ; 32.4]$ & $32.8[19.6 ; 62.2]$ & $20.1[13.8 ; 27.2]$ & $25.8[16.4 ; 37.2]^{*}$ & $22.9[14.0 ; 29.8]$ & $20.3[13.9 ; 27.3]$ \\
\hline $32.7[23.2 ; 57.1] \mathrm{T}$ & $62.5[31.6 ; 102.9]$ & $103.4[33.5 ; 151.1]$ & $50.3[29.3 ; 86.6]$ & $60.4[29.5 ; 103.4]$ & $41.5[31.3 ; 91.1]$ & $56.7[28.7 ; 89.7]$ \\
\hline $2.83[2.03 ; 3.44] \mathrm{T}$ & $3.61[2.46 ; 4.96]$ & $4.80[3.44 ; 5.79]$ & $3.27[2.40 ; 4.43]$ & $3.90[3.06 ; 5.21]$ & $3.36[2.37 ; 4.38]$ & $3.37[2.47 ; 4.62]$ \\
\hline $9.77 \pm 0.54$ & $9.73 \pm 0.99$ & $9.82 \pm 0.46$ & $9.76 \pm 0.64$ & $10.00 \pm 0.59$ & $9.82 \pm 0.66$ & $9.70 \pm 0.48$ \\
\hline $3.20 \pm 0.67$ & $3.04 \pm 0.73$ & $3.41 \pm 0.50$ & $3.18 \pm 0.68$ & $2.68 \pm 0.58 \mathrm{~T}$ & $3.06 \pm 0.70$ & $3.29 \pm 0.62$ \\
\hline $36 \pm 15$ & $36 \pm 14$ & $19 \pm 7 *$ & $37 \pm 14$ & $29 \pm 12 \Phi$ & $36 \pm 14$ & $35 \pm 15$ \\
\hline $23.5 \pm 2.9$ & $22.9 \pm 2.9$ & $21.1 \pm 4.9$ & $23.3 \pm 2.9$ & $21.3 \pm 2.3 \Phi$ & $22.9 \pm 2.8$ & $23.2 \pm 3.4$ \\
\hline $1.69 \pm 0.25$ & $1.67 \pm 0.23$ & $1.63 \pm 0.28$ & $1.69 \pm 0.23$ & $1.56 \pm 0.22 \Phi$ & $1.66 \pm 0.24$ & $1.73 \pm 0.22$ \\
\hline $1.0[0.5,1.4]$ & $1.0[0.6,1.5]$ & $1.4[0.8,1.8]$ & $1.0[0.6,1.4]$ & $1.3[0.8,1.6]$ & $1.0[0.6,1.5]$ & $1.1[0.6,1.4]$ \\
\hline $32 \pm 9$ & $34 \pm 13$ & $32 \pm 8$ & $32 \pm 11$ & $40 \pm 14 T$ & $34 \pm 12$ & $32 \pm 10$ \\
\hline $627[468,911]$ & $558[440,768]$ & $493[208,631]$ & $594[461,805]$ & $457[372,590] \Phi$ & $582[457,799]$ & $548[654,1041]$ \\
\hline $1.9[1.2,2.4]$ & $2.1[1.0,3.3]$ & $2.1[1.2,2.4]$ & $2.0[1.0,2.7]$ & $2.4[1.3,4.1]$ & $2.2[1.2,3.1]$ & $1.4[0.5,2.1] \Phi$ \\
\hline $84[51,103]$ & $80[59,100]$ & $80[24,112]$ & $83[59,105]$ & $70[44,92]^{*}$ & $79[57,98]$ & $94[68,124] \Phi$ \\
\hline
\end{tabular}

Age, yrs

Gender male, $\mathrm{y} / \mathrm{n}$

BMI, $\mathrm{kg} / \mathrm{m}^{2}$

Dialysis vintage, mo

Diabetes mellitus, \%

Cumulative steroids, $g$

eGFR, $\mathrm{ml} / \mathrm{min} / \mathrm{m}^{2}$

PTH, $\rho g / \mathrm{mL}$

PTH, xUNL

Total AP, U/L

BsAP, $\mu \mathrm{g} / \mathrm{L}$

Intact PINP, ng/mL

TRAP5b, U/L

Total calcium, mg/dL

Phosphate, mg/dL

Calcidiol, $\mu \mathrm{g} / \mathrm{L}$

Bicarbonate, $\mathrm{mmol} / \mathrm{L}$

Magnesium, mg/dL

24hr FECa, \%

24hr FEPO4, \%

Sclerostin, $\rho g / \mathrm{mL}$

sRANKL, $\rho g / m L$

OPG, $\rho g / \mathrm{mL}$

Mineralization

Volume

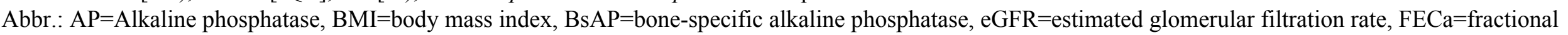
excretion of calcium, FEPO =fractional excretion of phosphate, $\mathrm{OPG}=$ osteoprotegerin, $\mathrm{PINP}=$ pro-collage type I pro-peptide, $\mathrm{PTH}=\mathrm{Parathyroid}$ hormone, $\mathrm{sRANKL}=$ soluble receptor activator of nuclear factor- $\mathrm{kB}$ ligand, TRAP $5 \mathrm{~b}=$ tartrate resistant acid phosphatase type $5 \mathrm{~b}$, $\mathrm{xUNL}=$ times $\mathrm{upper}$ normal limit 
Table 3. Demographic variables and bone turnover, mineralization, and volume by transiliac bone biopsy in patients with and without osteoporosis by dual energy $x$-ray absorptiometry at different skeletal sites

\begin{tabular}{|c|c|c|c|c|c|c|c|c|}
\hline & \multicolumn{2}{|c|}{ Lumbar spine } & \multicolumn{2}{|c|}{ Femoral neck } & \multicolumn{2}{|c|}{ Distal 1/3 radius } & \multicolumn{2}{|c|}{ Ultradistal radius } \\
\hline & $\begin{array}{l}T \text {-score } \geq-2.5 \\
\quad(n=84)\end{array}$ & $\begin{array}{l}\text { Osteoporosis } \\
\quad(n=24)\end{array}$ & $\begin{array}{c}T \text {-score } \geq-2.5 \\
(n=79)\end{array}$ & $\begin{array}{l}\text { Osteoporosis } \\
\quad(n=27)\end{array}$ & $\begin{array}{c}T \text {-score } \geq-2.5 \\
(n=62)\end{array}$ & $\begin{array}{l}\text { Osteoporosis } \\
\quad(n=29)\end{array}$ & $\begin{array}{c}T \text {-score } \geq-2.5 \\
(n=49)\end{array}$ & $\begin{array}{c}\text { Osteoporosis } \\
\quad(n=42)\end{array}$ \\
\hline Age, yrs & $55 \pm 12$ & $60 \pm 11 *$ & $55 \pm 12$ & $61 \pm 9.5 \Phi$ & $55 \pm 11$ & $60 \pm 12 *$ & $54 \pm 12$ & $61 \pm 10 \Phi$ \\
\hline Male gender & $63(75 \%)$ & $13(54 \%)^{*}$ & $53(67 \%)$ & $21(78 \%)$ & $42(68 \%)$ & $20(69 \%)$ & $26(53 \%)$ & $36(86 \%) \Phi$ \\
\hline $\begin{array}{l}\text { Body mass index, } \\
\mathrm{kg} / \mathrm{m}^{2}\end{array}$ & $25.7 \pm 4.9$ & $23.4 \pm 3.9 \Phi$ & $25.7 \pm 5.1$ & $23.7 \pm 3.6^{*}$ & $25.4 \pm 5.2$ & $24.3 \pm 4.7$ & $26.4 \pm 5.2$ & $23.5 \pm 4.3 \mp$ \\
\hline Dialysis vintage, mo & $22[10,35]$ & $31[19,55] \mathrm{T}$ & $24[10,36]$ & $31[15,45]$ & $21[8,32]$ & $33[14,54] \mathrm{T}$ & $21[8,35]$ & $31[13,47]$ \\
\hline Diabetes mellitus, \% & $29(35 \%)$ & $7(29 \%)$ & $26(33 \%)$ & $10(37 \%)$ & $22(36 \%)$ & $7(24 \%)$ & $12(25 \%)$ & $17(41 \%)$ \\
\hline \multirow[t]{2}{*}{ Cumulative steroids, $\mathrm{g}$} & $2.4[1.8 ; 2.8]$ & $2.3[1.6 ; 3.1]$ & $2.3[1.8 ; 2.9]$ & $2.4[1.9 ; 2.8]$ & $2.4[1.8 ; 3.0]$ & $2.2[1.6 ; 2.8]$ & $2.4[1.8 ; 2.9]$ & $2.3[1.8 ; 2.8]$ \\
\hline & \multicolumn{6}{|c|}{$\underline{T M V \text { classification }}$} & & \\
\hline \multicolumn{9}{|l|}{ Turnover } \\
\hline Low & $17(20 \%)$ & $6(25 \%)$ & $18(23 \%)$ & $4(15 \%)$ & $14(23 \%)$ & $5(17 \%)$ & $9(18 \%)$ & $10(24 \%)$ \\
\hline Normal & $65(77 \%)$ & $17(71 \%)$ & $59(75 \%)$ & $22(82 \%)$ & $47(76 \%)$ & $22(76 \%)$ & $39(80 \%)$ & $30(71 \%)$ \\
\hline High & $2(2 \%)$ & $1(4 \%)$ & $2(3 \%)$ & $1(4 \%)$ & $1(2 \%)$ & $2(7 \%)$ & $1(2 \%)$ & $2(5 \%)$ \\
\hline \multicolumn{9}{|l|}{ Mineralization } \\
\hline Normal & $71(85 \%)$ & $23(96 \%)$ & $67(85 \%)$ & $25(93 \%)$ & $52(84 \%)$ & $28(97 \%)$ & $42(86 \%)$ & $38(91 \%)$ \\
\hline Delayed & $13(15 \%)$ & $1(4 \%)$ & $12(15 \%)$ & $2(7 \%)$ & $10(16 \%)$ & $1(3 \%)^{*}$ & $7(14 \%)$ & $4(10 \%)$ \\
\hline \multicolumn{9}{|l|}{ Volume } \\
\hline$\overline{\text { Low }}$ & $12(14 \%)$ & $9(38 \%) \Phi$ & $11(14 \%)$ & $9(33 \%) \Phi$ & $10(16 \%)$ & $9(31 \%)^{*}$ & $9(18 \%)$ & $10(24 \%)$ \\
\hline Normal & $72(86 \%)$ & $15(62 \%)$ & $68(86 \%)$ & $18(67 \%)$ & $52(84 \%)$ & $20(69 \%)$ & $40(82 \%)$ & $32(76 \%)$ \\
\hline
\end{tabular}

Data are mean $\pm \mathrm{SD}$, median[IQR], or $n(\%)$ with $*=\mathrm{p}<0.10$ and $\Phi=\mathrm{p}<0.05$ by Student's $t$ test, Wilcoxon rank-sum test, or Pearson's $X^{2}$ test, respectively 
Table 4. Bone histomorphometry in different categories of mineral metabolism disturbances after kidney transplantation

\begin{tabular}{|c|c|c|c|c|c|c|c|c|}
\hline & $\begin{array}{c}\text { PTH } \leq 1.5 \\
\text { xUNL }(n=71)\end{array}$ & $\begin{array}{c}\text { PTH }>1.5 \\
\text { xUNL }(n=67)\end{array}$ & $\begin{array}{c}\mathrm{Ca} \leq 10.3 \mathrm{mg} / \mathrm{dL} \\
(n=114)\end{array}$ & $\begin{array}{c}\mathrm{Ca}>10.3 \\
\mathrm{mg} / \mathrm{dL}(n=26)\end{array}$ & $\begin{array}{c}\mathrm{PO}_{4} \geq 2.3 \mathrm{mg} / \mathrm{dL} \\
(n=123)\end{array}$ & $\begin{array}{c}\mathrm{PO}_{4}<2.3 \\
\mathrm{mg} / \mathrm{dL}(n=18)\end{array}$ & $\begin{array}{c}\text { Calcidiol } \geq 30 \\
\mathrm{ng} / \mathrm{mL}(n=85)\end{array}$ & $\begin{array}{c}\text { Calcidiol }<30 \\
\mathrm{ng} / \mathrm{mL}(n=53)\end{array}$ \\
\hline $\begin{array}{l}\mathrm{BFR} / \mathrm{TAr} \\
\mu^{2} / \mathrm{mm}^{2} / \text { day }\end{array}$ & $110[40 ; 198]$ & $185[66 ; 372] \mathrm{T}$ & $130[44 ; 221]$ & $193[36 ; 309]$ & 134 [44; 229] & $208[57 ; 496]$ & $138[42 ; 229]$ & $138[49 ; 278]$ \\
\hline $\mathrm{MPm} / \mathrm{BPm}, \%$ & $4.4[2.5,7.7]$ & $8.6[3.1,12.7] \mathrm{T}$ & $5.0[2.7,9.6]$ & $8.7[2.4,12.5]$ & $5.4[2.7,9.9]$ & $7.4[3.1,14.9]$ & $5.9[2.8,9.7]$ & $4.7[2.6,10.7]$ \\
\hline Mlt, days & $29[20 ; 51]$ & $46[22 ; 78] \Phi$ & $32[20 ; 65]$ & $46[21 ; 80]$ & $33[20 ; 59]$ & $55[30 ; 86]^{*}$ & $33[21 ; 57]$ & $46[20 ; 80]$ \\
\hline $\mathrm{ObPm} / \mathrm{BPm}, \%$ & $1.2[0.0 ; 3.3]$ & $6.6[1.2 ; 13.4] \mathrm{T}$ & $2.0[0.0 ; 7.5]$ & $2.9[0.9 ; 7.5]$ & $2.1[0.5 ; 7.3]$ & $3.6[0.9 ; 10.9]$ & $2.7[0.6 ; 7.2]$ & $1.5[0.0 ; 12.0]$ \\
\hline $\mathrm{OcPm} / \mathrm{BPm}, \%$ & $0.1[0.0 ; 0.6]$ & $0.6[0.3 ; 1.2] \mathrm{T}$ & $0.4[0.0 ; 0.9]$ & $0.6[0.2 ; 0.9]$ & $0.4[0.0 ; 0.9]$ & $0.5[0.3 ; 0.9]$ & $0.4[0.0 ; 1.0]$ & $0.5[0.0 ; 0.8]$ \\
\hline $\mathrm{EPm} / \mathrm{BPm}, \%$ & $1.6[0.6 ; 3.3]$ & $2.6[1.3 ; 4.3] \mp$ & $2.2[0.9 ; 3.6]$ & $3.4[1.4 ; 4.5] \Phi$ & $2.2[1.0 ; 3.9]$ & $2.5[1.3 ; 3.5]$ & $2.3[1.0 ; 3.9]$ & $2.3[1.0 ; 3.9]$ \\
\hline $\mathrm{OAr} / \mathrm{BAr}, \%$ & $1.4[0.8 ; 2.8]$ & $3.5[1.9 ; 7.0] \mp$ & $2.0[0.9 ; 4.4]$ & $3.1[1.3 ; 6.4]$ & $2.0[1.0 ; 4.3]$ & $4.2[2.2 ; 8.5] \mathrm{T}$ & $2.0[0.9 ; 4.2]$ & $3.1[1.2 ; 6.1]$ \\
\hline $\mathrm{OPm} / \mathrm{BPm}, \%$ & $\begin{array}{c}13.3 \\
{[9.0 ; 21.0]}\end{array}$ & $\begin{array}{c}31.1 \\
{[18.3 ; 47.9] \mp}\end{array}$ & $\begin{array}{c}17.7 \\
{[10.0 ; 31.2]}\end{array}$ & $\begin{array}{c}24.7 \\
{[13.5 ; 42.4]}\end{array}$ & $\begin{array}{c}16.6 \\
{[10.1 ; 30.5]}\end{array}$ & $\begin{array}{c}33.5 \\
{[18.9 ; 49.5] \mp}\end{array}$ & $\begin{array}{c}15.7 \\
{[10.0 ; 29.2]}\end{array}$ & $\begin{array}{c}24.8 \\
{[10.8 ; 38.6]}\end{array}$ \\
\hline $\mathrm{OWi}, \mu \mathrm{m}$ & $6.6[5.4 ; 9.3]$ & $8.0[5.9 ; 10.0]$ & $6.9[5.4 ; 9.4]$ & $7.5[6.2 ; 10.6]$ & $6.9[5.4 ; 9.4]$ & $8.7[6.2 ; 10.8]$ & $6.6[5.3 ; 9.3]$ & $7.6[6.1 ; 10.7] \mathrm{T}$ \\
\hline BAr/TAr, $\%$ & $20.0 \pm 6.5$ & $20.2 \pm 5.8$ & $20.3 \pm 6.5$ & $19.5 \pm 4.9$ & $20.0 \pm 6.3$ & $21.6 \pm 5.2$ & $19.6 \pm 6.1$ & $20.9 \pm 6.3$ \\
\hline \multicolumn{9}{|c|}{ Data are mean $\pm \mathrm{SD}$, median[IQR], or $n[\%)$ with $*=p<0.10$ and $\Phi=p<0.05$ by Student's $t$ test, Wilcoxon rank-sum test, or Pearson's $X^{2}$ test, respectively } \\
\hline \multicolumn{9}{|c|}{ 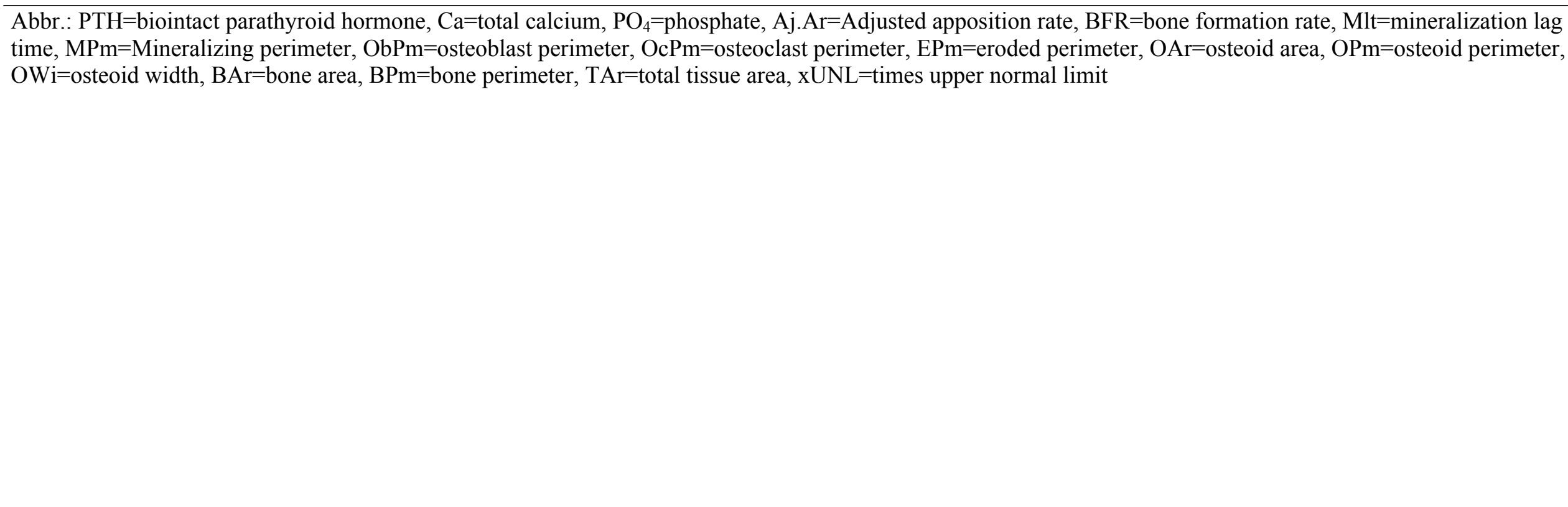 } \\
\hline
\end{tabular}




\section{Legends to figures}

Figure 1: Disturbances of bone turnover, mineralization, and volume by transiliac bone biopsy 1 year after kidney transplantation

Figure 2: Osteoporosis ( $T$-score $<-2.5)$ and osteopenia $(T$-score $<-1.0$ and $\geq-2.5)$ by Dual Energy X-ray Absorptiometry at different skeletal sites 1 year after kidney transplantation

Figure 3: Disturbances of mineral metabolism 1 year after kidney transplantation; hyperparathyroidism (biointact parathyroid hormone, PTH $>1.5 \mathrm{x}$ upper normal limit), hypophosphatemia (phosphate, PO4 $<2.3$ $\mathrm{mg} / \mathrm{dL}$ ), and hypercalcemia (total calcium, $\mathrm{Ca}>10.3 \mathrm{mg} / \mathrm{dL}$ ) 


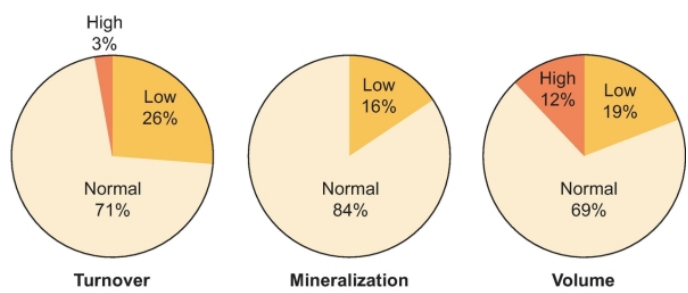




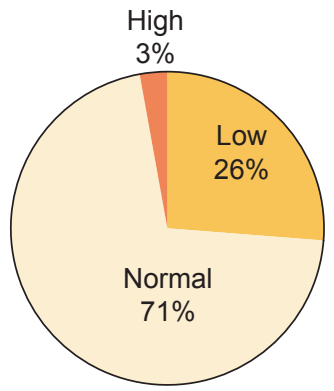

Turnover

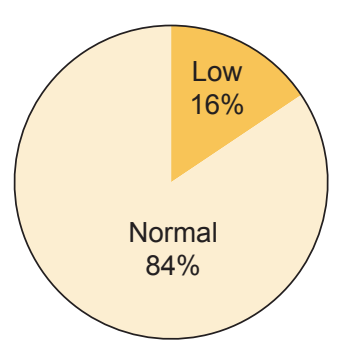

Mineralization

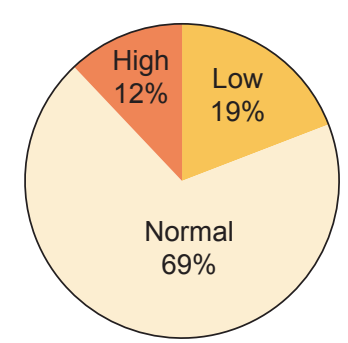

Volume

NDT-00674-2021.R1-fig1 


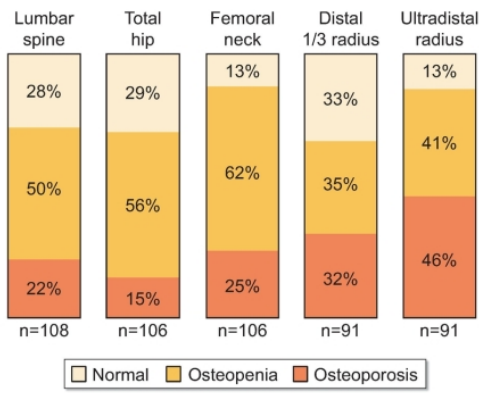

NDT-00674-2021.R1-fig2

$85 \times 249 \mathrm{~mm}(300 \times 300 \mathrm{DPI})$ 


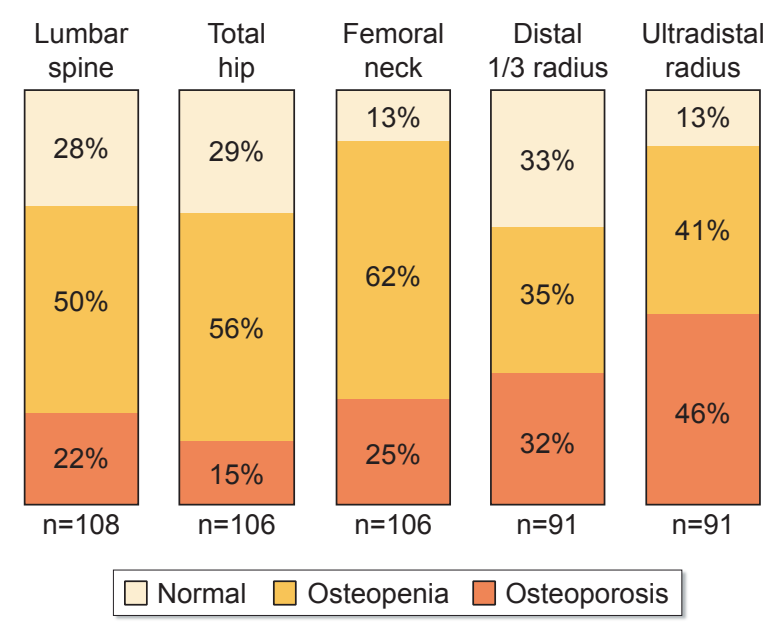




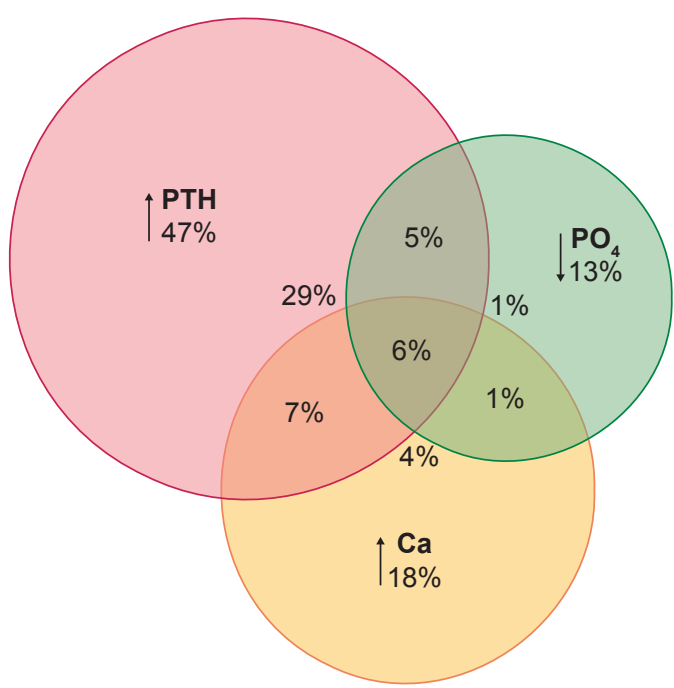

